\title{
7×100 Gbps PAM-4 Transmission over 1-km and 10-km Single Mode 7-core Fiber using 1.5- $\mu \mathrm{m}$ SM-VCSEL
}

\author{
Xiaodan Pang ${ }^{1,3}$, Joris Van Kerrebrouck ${ }^{2}$, Oskars Ozolins ${ }^{3}$, Rui Lin ${ }^{1,4}$, Aleksejs Udalcovs ${ }^{3}$, Lu Zhang, \\ Silvia Spiga ${ }^{5}$, Markus C. Amann ${ }^{5}$, Geert Van Steenberge ${ }^{6}$, Lin $\mathrm{Gan}^{4}$, Ming Tang ${ }^{4}$, Songnian $\mathrm{Fu}^{4}$, \\ Richard Schatz ${ }^{1}$, Gunnar Jacobsen ${ }^{3}$, Sergei Popov ${ }^{1}$, Deming Liu ${ }^{4}$, Weijun Tong ${ }^{7}$, Guy Torfs ${ }^{2}$, \\ Johan Bauwelinck ${ }^{2}$, Xin Yin ${ }^{2}$ and Jiajia Chen ${ }^{1}$ \\ ${ }^{1}$ KTH Royal Institute of Technology, Kista, Sweden \\ ${ }^{2} I D L a b$, INTEC, Ghent University - imec, Gent, Belgium \\ ${ }^{3}$ Networking and Transmission Laboratory, RISE Acreo AB, Kista, Sweden \\ ${ }^{4}$ Huazhong University of Science and Technology, Wuhan, China \\ ${ }^{5}$ Walter Schottky Institut, Technische Universität München, Garching, Germany \\ ${ }^{6} \mathrm{CMST}$, Ghent University - imec, Gent, Belgium \\ ${ }^{7}$ Yangtze Optical fiber and Cable Joint Stock Limited Company, Wuhan, China \\ Email: jiajiac@kth.se; xin.yin@ugent.be
}

\begin{abstract}
Gbps} / \lambda$ /core PAM-4 transmission is successfully demonstrated over 1-km and 10$\mathrm{km}$ single mode 7 -core fiber links, enabled by directly modulated 1.5 - $\mu \mathrm{m}$ single mode VCSEL of $23 \mathrm{GHz}$ modulation bandwidth with pre- and post- digital equalizations.

OCIS codes: (060.4510) Optical communications; (250.7260) Vertical cavity surface emitting lasers; (200.4650) Optical interconnects
\end{abstract}

\section{Introduction}

Current demand for supporting high-performance computing (HPC) and datacenter applications has been bringing highly stringent requirements on short-reach transmission techniques, in terms of data rate, cost, reliability, power consumption and footprint, simultaneously. Vertical cavity surface emitting laser (VCSEL)-based transceivers are considered as promising candidates to well match such requirements, particularly owing to the merit for highdensity integration [1]. Furthermore, VCSEL array can seamlessly extend its potential to a relatively longer-term evolution towards "parallelism", enabled by space division multiplexing (SDM) techniques, so as to scale up the lane count per fiber and reduce cabling complexity [2]. To approach this vision, many efforts have been put into research and demonstrations in several aspects with short wavelength multimode (MM)-VCSELs, including advanced modulation formats such as pulse amplitude modulation (PAM), discrete-multi-tone (DMT) and multiband approach of carrierless amplitude phase (MultiCAP), supporting high-speed single lane transmissions [3-5], as well as coupling MM-VCSEL array with multicore fibers (MCFs) for high aggregate data rate [6, 7]. For applications requiring distances more than a few kilometers, longer wavelength single mode (SM)-VCSELs with single mode fiber (SMF) links are more feasible. A few recent works on high data rate transmission with $1.5-\mu \mathrm{m}$ SM-VCSELs have also shown promising results towards implementation $[8,9]$.

In this work, we report on a demonstration of high-speed PAM-4 transmission using 1.5- $\mu \mathrm{m}$ SM-VCSEL over 7core fiber links with different distances. We have successfully generated up to 70 Gbaud PAM-4 signal with the directly modulated VCSEL, and transmitted 50 Gbaud PAM-4 over 1-km dispersion-uncompensated and 10-km dispersion-compensated single mode MCF links. With pre-equalization based on accurate end-to-end channel characterization, combined with low-complexity digital post-equalization, bit-error-rate (BER) performances over all the cores are achieved below the 7\% overhead-hard decision forward error correction (7\% OH-HDFEC) limit.

\section{Experimental setup}

Figure 1 shows our experimental setup, including the pictures of the probe station and top view of the VCSEL chip. The L-I-V characteristic of the VCSEL is shown as inset. The threshold current is around $1.5 \mathrm{~mA}$ and an optimal current is found to be $\sim 8 \mathrm{~mA}$, whereafter the VCSEL output power degrades due to saturation. Figure 2 (a) shows the modulation frequency response of the employed VCSEL chip. The maximum 3-dB modulation bandwidth is measured to be $\sim 23 \mathrm{GHz}$. The PAM-4 symbols are offline generated from a pseudorandom bit sequence of word length $2^{15}-1$ (PRBS-15), before being up-sampled and filtered with a raise cosine filter of 0.15 roll-off factor. Frequency domain channel pre-equalization is performed based on the characterized end-to-end channel frequency response as shown in Fig. 2 (a)-(c). After pre-equalization the signal is resampled and loaded to a $92 \mathrm{GSa} / \mathrm{s}$ arbitrary waveform generator (AWG) to drive the VCSEL. The amplitude of the driving signal is set to be $700 \mathrm{mVpp}$. The VCSEL is operated at room temperature without active cooling, and the optimal driving current is found to be $7.8 \mathrm{~mA}$, considering both bandwidth and output power. 


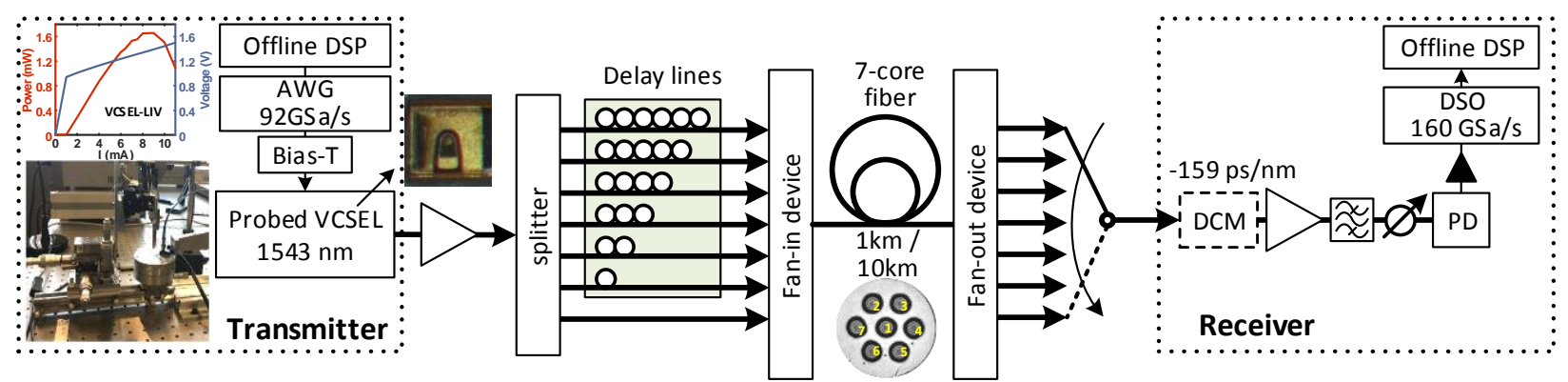

Fig. 1. Experimental setup. VCSEL L-I-V curves, photos of probe station, VCSEL chip and MCF shown as insets.

The VCSEL output is amplified and splitted into seven lanes with proper decorrelations between them, to emulate parallel independent channels entering the fan-in (FI) device for the 7-core fiber. The MCF used in this experiment has a $150-\mu \mathrm{m}$ cladding diameter and $42-\mu \mathrm{m}$ core pitch, with inter-core crosstalk of $\sim-45 \mathrm{~dB} / 100 \mathrm{~km}$, attenuation of $0.20 \mathrm{~dB} / \mathrm{km}$ and dispersion coefficient of $17.1 \mathrm{ps} / \mathrm{nm} / \mathrm{km}$. After transmission over the 1-km or 10-km MCF link, a fan-out (FO) device is used to couple the signals to single-core fibers, prior to the receiver. This process in practice should be more effectively achieved with a VCSEL/PD array butt-coupled to each core of the MCF [2]. For 1-km MCF transmission the link is uncompensated, and a fixed dispersion compensation module (DCM) of $159 \mathrm{ps} / \mathrm{nm}$ is used for the 10-km MCF case, yielding a residual dispersion of $\sim 16 \mathrm{ps} / \mathrm{nm}$. As one can see from Fig. 2(b), only slight differences are found between the responses of each core of the 1-km MCF, indicating approximate homogeneous characteristics of the cores. More pronounced differences between the cores can be observed after the 10-km MCF with the DCM, as shown in Fig. 2(c). We attribute this to the filtering effect induced by the FI/FO devices, as well as slight shifted chromatic dispersion coefficients between cores. The 3-dB bandwidth of all the channels after both MCF links are considerably reduced compared with the back-to-back case, determined by the RF fading induced by the VCSEL chirp and dispersion interaction.
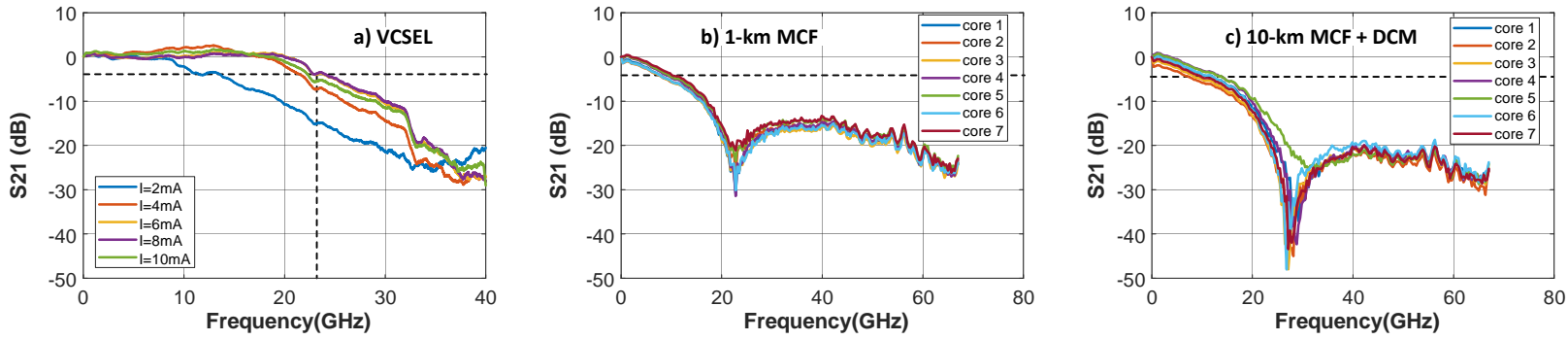

Fig.2. S21 response of (a) the VCSEL chip, and after transmission of each core of the (b) 1-km MCF and (c) 10-km MCF + DCM.

At the receiver, we detect and evaluate each FO output signal individually with a 90-GHz PIN photodiode (PD). The electrical signal is amplified by an 11-dB linear amplifier, sampled at a $160 \mathrm{GSa} / \mathrm{s}$ real-time digital storage oscilloscope (DSO) and processed offline. The offline processing routine consists of a low-pass filter, a maximum variance-based timing recovery, a symbol-spaced decision-feedback equalizer (DFE) and an error counter.

\section{Results}

Figure 3 shows the B2B BER curves for the modulated PAM-4 signal of different baud rates. BER performance of below the 7\% OH-HDFEC limit of $3.8 \times 10^{-3}$ is successfully achieved at $\sim 0 \mathrm{dBm}$ received optical power (RoP) for up to 70 Gbaud PAM-4 signal, with DFE of 5 feedforward (FF) and 5 feedback (FB) taps. This is mainly owning to the effective channel pre-equalization at the transmitter. For the 50, 56 and 64 Gbaud signals, DFE with only 3-FF + 3FB taps are sufficient to equalize the residual inter-symbol interference (ISI). The eye diagrams after equalization at RoP of $7 \mathrm{dBm}$ are also shown in Fig. 3, and clear eye opening can be seen for all tested cases, despite of the observed slight eye skew caused by signal dependent rise/fall time for directly modulated VCSEL [10].

Finally, Fig. 4 shows the BER performance after the 1-km and 10-km MCF transmissions for PAM-4 signal at 50 Gbaud. The reduced baud rate after the MCFs compared with B2B case is mainly due to the reduced 3-dB channel bandwidth, as already shown in Fig. 2. For the uncompensated 1-km MCF cases, a DFE of 7-FF-tap+7-FBtap can successfully recover the received signals in all the cores to reach BER performance below the 7\%-OHHDFEC limit. For the 10-km dispersion-compensated MCF cases, due to slight relaxed bandwidth limit, a DFE of 3FF-tap+3-FB-tap is shown to be sufficient. Additionally, different performances between different cores can be seen in both cases. Selected eye diagrams of the best and worst cores in each case are also shown. Such performance differences are in accordance with the differences in the characterized frequency response. It should be noted that 

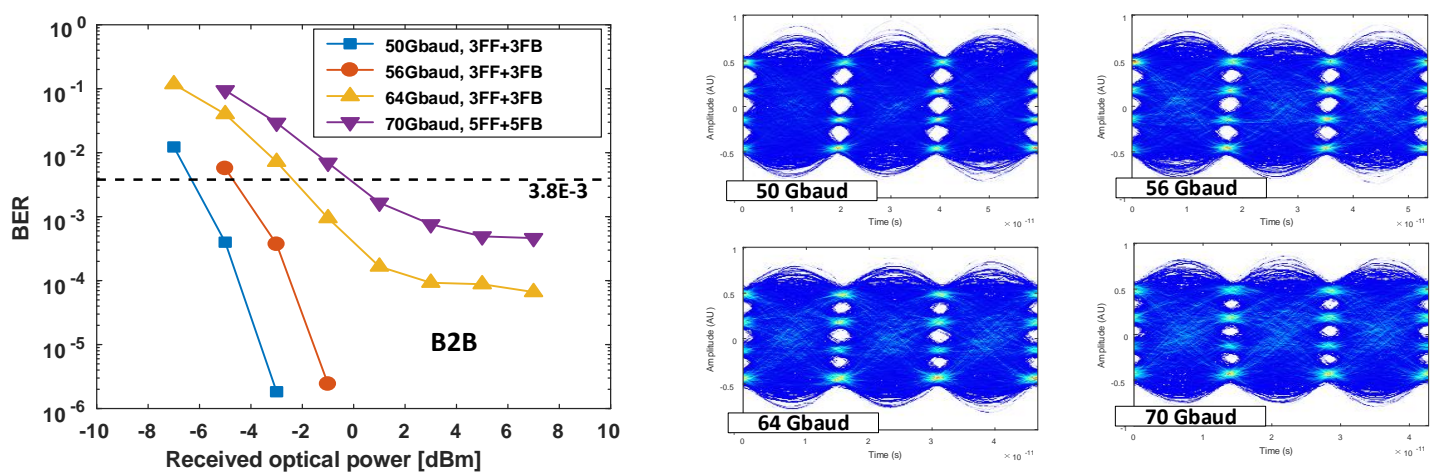

Fig. 3. B2B BER results for up to 70 Gbaud PAM-4 and equalized eye diagrams at $7 \mathrm{dBm}$ RoP.

due to added complexity, we did not apply any specific amplitude-level dependent symbol decision method or nonlinear equalization techniques to mitigate the eye skew. Therefore, due to narrower horizontal eye opening, better BER performance and/or higher baud rates can be potentially achieved after eye skew correction [10].
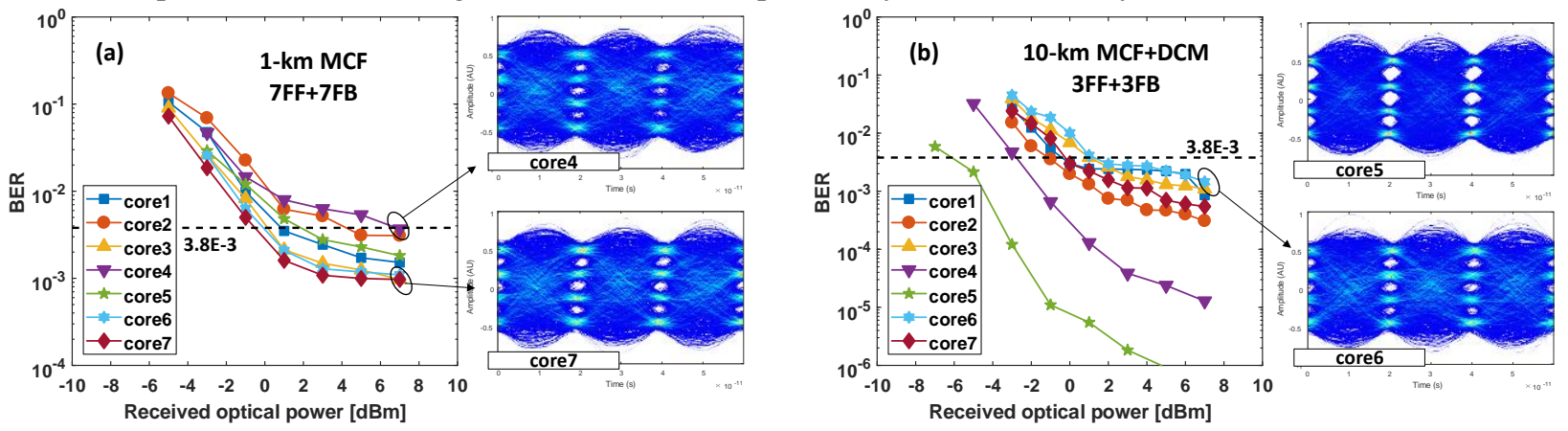

Fig. 4. BER results for 50 Gbaud PAM-4 signal after 1-km and 10-km MCF and eye diagrams at $7 \mathrm{dBm}$ RoP for the best and worst cores.

\section{Conclusion}

We have experimentally demonstrated high-speed short-reach optical transmissions with long-wavelength SMVCSEL and MCF. Up to 70 Gbaud PAM-4 signal is generated with the directly modulated VCSEL, and we have successfully achieved up to 50 Gbaud PAM-4 over 1-km dispersion-uncompensated and 10-km dispersioncompensated transmission through all the cores of the MCF links. Pre-equalization based on end-to-end link characterization together with simple post-equalization techniques prove to be sufficient for this configuration, and further improvement in terms of data rate can be expected with effective eye skew correction methods.

\section{Acknowledgement}

This work was supported by the Swedish Research Council (VR), the Swedish Foundation for Strategic Research (SSF), Göran Gustafsson Foundation, Swedish ICT TNG, FP7 project MIRAGE (ref.318228), H2020 project WIPE (ref.688572), Vinnova funded Celtic Plus sub-project C2015/3-5 SENDATE-FICUS, Natural Science Foundation of Guangdong Province and National Natural Science Foundation of China.

\section{References}

[1] H. Nasu et al., ">1-Tb/s on-board optical engine for high-density optical interconnects," W1A.4, OFC 2017.

[2] D. Kuchta, "High-capacity VCSEL links," Tu3C.4, OFC 2017.

[3] F. Karinou et al., "112 Gb/s PAM-4 optical signal transmission over 100-m OM4 multimode fiber for high-capacity data-center interconnects," M2C.2, ECOC 2016.

[4] C. Kottke et al., "High speed $160 \mathrm{~Gb} / \mathrm{s}$ DMT VCSEL transmission using pre-equalization," W4I.7, OFC 2017.

[5] R. Puerta et al., "107.5 Gb/s $850 \mathrm{~nm}$ multi- and single-mode VCSEL transmission over 10 and $100 \mathrm{~m}$ of multi-mode fiber," Th5B.5, OFC 2016.

[6] B. G. Lee et al., "End-to-end multicore multimode fiber optic link operating up to $120 \mathrm{~Gb} / \mathrm{s}$," J. Lightw. Technol. 30(6), 886-892 (2012).

[7] P. Westbergh et al., "VCSEL arrays for multicore fiber interconnects with an aggregate capacity of $240 \mathrm{~Gb} / \mathrm{s}$," IEEE Photon. Technol. Lett. 27(3), 296-299 (2015).

[8] N. Eiselt et al., "Experimental demonstration of $56 \mathrm{Gbit} / \mathrm{s}$ PAM-4 over $15 \mathrm{~km}$ and $84 \mathrm{Gbit} / \mathrm{s}$ PAM-4 over $1 \mathrm{~km}$ SSMF at $1525 \mathrm{~nm}$ using a 25G VCSEL," Th.1.C.1, ECOC 2016.

[9] C. Xie et al., "Single-VCSEL 100-Gb/s short-reach system using discrete multi-tone modulation and direct detection," Tu2H.2, OFC 2015. [10] J. M. Castro et al., "Eye skew modeling, measurements and mitigation methods for VCSEL PAM-4 channels at data rates over $66 \mathrm{~Gb} / \mathrm{s}$," W3G.3, OFC 2017. 